\title{
Change in Bone Mineral Density of Filipino Patients with Osteogenesis Imperfecta after 6 Months of Pamidronate Therapy in a Tertiary Hospital in the Philippines
}

\author{
Mary Ann R. Abacan, ${ }^{1,2}$ Ryan Albert Leonard N. Boquiren, ${ }^{3}$ Imarzen V. Elepaño ${ }^{3}$ \\ and Maria Melanie Liberty B. Alcausin ${ }^{1,2}$ \\ ${ }^{1}$ Section of Genetics, Department of Pediatrics, College of Medicine and Philippine General Hospital University of the Philippines Manila \\ 2Institute of Human Genetics, National Institutes of Health, University of the Philippines Manila \\ ${ }^{3}$ Department of Radiology, College of Medicine and Philippine General Hospital, University of the Philippines Manila
}

\begin{abstract}
Background. Osteogenesis imperfecta is a heritable disorder due to a collagen gene mutation causing a structural abnormality leading to brittle bones and osteopenia. To address the osteopenia, intravenous bisphosphonates (pamidronate) act by temporarily halting the action of osteoclasts giving time for osteoblasts to build bone. To date, there has been no local data regarding the improvement in bone mineral density of Filipino patients with osteogenesis imperfecta following treatment.

Methods. This study is a retrospective review that included six patients aged 1 year and 10 months- 9 years and 9 months old at the Philippine General Hospital with moderate to severe osteogenesis imperfecta who have undergone six months of pamidronate infusions at $1 \mathrm{mg} / \mathrm{kg} /$ dose monthly or a total dose of $6 \mathrm{mg} / \mathrm{kg}$. Chart review was done. Hand radiographs taken at baseline and after six months of therapy were reviewed by a radiologist who was blinded, to determine metacarpal indices.
\end{abstract}

Results. There was an increasing trend in the metacarpal index from baseline to six months post-treatment with a mean difference of $0.053 \mathrm{~mm}(\mathrm{Cl}-0.0112$ to 0.117$)$. However, the increase was not statistically significant ( $p$ value 0.0874 ) when analyzed using the paired t-test at a $95 \%$ confidence interval. No adverse events were noted and only one patient reported a fracture after starting therapy.

Conclusion. Bisphosphonate infusions among the six pediatric patients with moderate to severe osteogenesis imperfecta are well tolerated and although the increase in the metacarpal index from baseline after six months of treatment is not statistically significant, the trend shows improvement of the osteopenia from baseline.

Key Words: osteogenesis imperfecta, bisphosphonate, bone mineral density

Presented as a poster at the 11th Asia Pacific Conference of Human Genetics, Sept. 16-18, 2015, Hanoi, Vietnam.

Corresponding author: Mary Ann R. Abacan, MD

Institute of Human Genetics

Room 203, 2nd Floor, NIH Building

University of the Philippines Manila

Telefax No: +632 5257459

Email: maabacan@post.upm.edu.ph

\section{Introduction}

Osteogenesis imperfecta (OI) or "brittle bone disease" is a heritable disorder caused by a mutation in the genes that code for pro-collagen, COL1A1 and COL1A2, ${ }^{1}$ which leads to an increase in osteoclastic activity and a reduction in formation of new bone, and is reflected as decrease in bone mass. ${ }^{1,2}$ To date, there are fifteen types of OI that have been identified according to clinical features and inheritance pattern. $^{3} \quad$ The widely used classification is that of Sillence and colleagues. ${ }^{4}$ Regardless of type, the clinical expression is primarily that of osteopenia, bone fragility, frequent fractures, progressive deformity, loss of mobility, and chronic bone pain. ${ }^{1,5}$

OI is the most common genetic cause of osteoporosis. ${ }^{6}$ The prevalence is reported at 6-7 per 100,000. ${ }^{7}$ According to the data available from the Philippine Osteogenesis Imperfecta Support Group, as of December 2014 there are 56 diagnosed patients in Luzon and 11 in the Visayas area. Since there is an absence of a formal national registry for OI, the true prevalence in the Philippines may be underestimated.

Based on the database of the Section of Genetics, Department of Pediatrics at the University of the Philippines-Philippine General Hospital (UP-PGH), there are 68 clinically diagnosed patients as of December 2014. However, only 41 are on regular follow-up. Of these 41,6 have OI type 1, 18 have type III, 11 have type IV, and 6 have type $\mathrm{V}$.

The definitive treatment for OI is gene therapy. Investigations are underway to the applicability of this therapy in humans. Medications seek to address the osteopenia that is present in osteogenesis imperfecta patients. The goal is to increase bone strength, either by increasing the amount of bone protein matrix or by increasing bone mineralization via a reduction in bone remodelling. ${ }^{8}$

Bisphosphonates are structural analogues of inorganic pyrophosphate. By specifically inhibiting osteoclast mediated bone-resorption, it presumptively allows boneforming osteoblasts more time to promote bone formation, 
albeit in the setting of abnormal collagen matrix as seen in patients with OI.9,10 The use of intravenous pamidronate therapy has been widely studied in patients with OI and is noted to reduce the incidence of fracture and increase bone mineral density, while reducing pain and increasing energy levels. ${ }^{1,2,8,11-17}$

A study by Alcausin et $\mathrm{al}^{18}$ reported that among 14 Filipino children with moderate to severe OI receiving intravenous pamidronate therapy, treatment was generally well tolerated and led to decrease in long bone fractures and improvement in vertebral shape. ${ }^{7}$ This study, however, did not include local data regarding the bone mineral density following treatment.

The best way to measure bone mineral density is through the use of dual energy x-ray absorptiometry (DEXA). Due to the prohibitive costs, patients are unable to undergo this procedure. A readily available and cheaper alternative is measurement of the metacarpal index. A good correlation has been found between metacarpal index and DEXA. ${ }^{19}$

This study seeks to determine the change in bone mineral density measured through metacarpal index after 6 months of intravenous bisphosphonate (pamidronate) treatment in patients with OI. Secondary parameters are to report the number of fractures sustained per month while on pamidronate therapy and to describe any adverse reactions to pamidronate therapy.

\section{Methods}

This is a retrospective study of six pediatric patients between the ages of 1 year and 10 months- 9 years and 9 months old diagnosed with moderate to severe OI seen at the UP-PGH from January 2006 to June 2011 who have undergone six months of pamidronate therapy or a total of $6 \mathrm{mg} / \mathrm{kg}$ and have available pre- and post-treatment radiographs. The diagnosis of osteogenesis imperfecta was made based on clinical and radiographic features. The study was reviewed and approved by the Ethical Review Board of the UP-PGH.

Indications for initiating pamidronate therapy include patients with Type III or severe OI and other types of osteogenesis imperfecta where there are recurrent long bone fractures, vertebral crush fractures, long bone deformity, bone pain or low bone mass. ${ }^{20}$ Patients are given a priming dose of $0.5 \mathrm{mg} / \mathrm{kg}$ before the intestive phase of therapy. During the intensive phase, pamidronate is given at a dose of $1 \mathrm{mg} / \mathrm{kg}$ monthly for the first six months then every two months at $1.5 \mathrm{mg} / \mathrm{kg} /$ dose for three cycles. If clinical improvement is noted after a year, the therapy may be given every two months at $1.5 \mathrm{mg} / \mathrm{kg} /$ dose for one year. After the second year of infusion, the maintenance phase is started. The dosage and duration of intervals depend on the patient's age.
Retrieval of the charts of the patients was done and baseline characteristics (age, sex, type of osteogenesis imperfecta, weight, height/length, Vitamin D levels, serum calcium, magnesium, phosphorus, alkaline phosphatase, creatinine, and BUN levels) were noted. Adverse events during treatment and fracture incidence (reported as fractures per month) were noted. Fracture incidence is defined as the number of fractures sustained in the six months prior to treatment initiation and throughout the treatment period.

The estimation of bone mineral density was done through the measurement of metacarpal index. ${ }^{21}$ Patients' left hand $\mathrm{x}$-rays taken from their first consult served as baseline or pre-treatment $x$-rays and their x-ray after the sixth month of treatment were retrieved. These radiographs are taken as a monitoring tool to assess response to treatment per pamidronate therapy protocol of the UP-PGH Section of Genetics. A total of sixteen radiograph films from seven patients were retrieved. The metacarpal indices were measured by a radiologist who was blinded to the identities of the patients.

The metacarpal index was computed as the ratio of the cortical thickness of the radial side plus the cortical thickness of the ulnar side and the outer diameter of the bone, specifically the $2^{\text {nd }}$ metacarpal of the left hand. It was also defined by the equation: outer cortical diameter - inner cortical diameter divided by the outer cortical diameter ${ }^{22}$ (Figure 1). The inner and outer diameters were measured at the midshaft location using a vernier calliper with a gradation of $0.05 \mathrm{~mm}$ in the vernier scale. Three measurement trials for each of the plates were done and recorded.

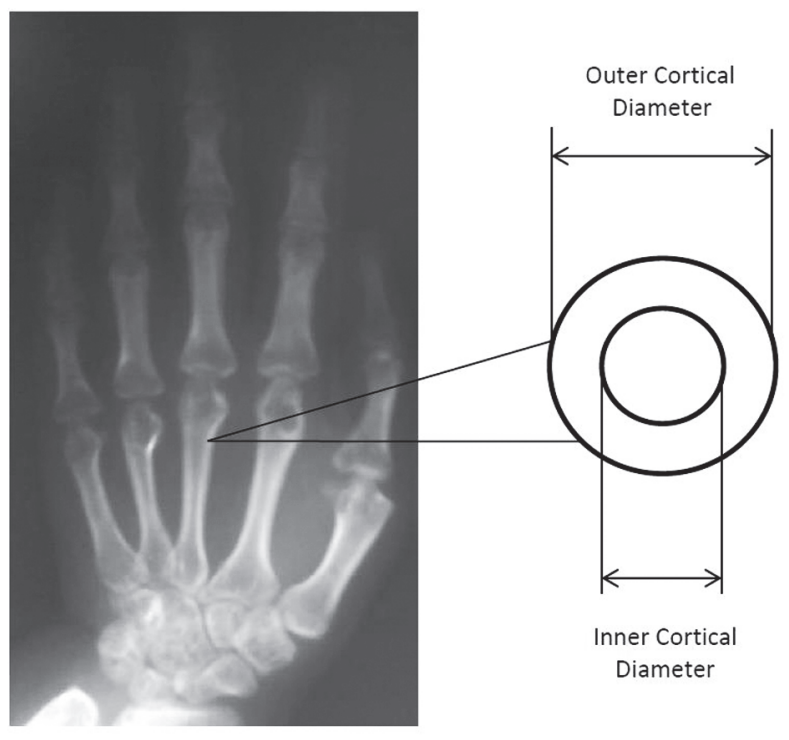

Figure 1. Measurement of the metacarpal index. 
The pre- and post-treatment metacarpal indices were analyzed using a paired t-test and estimated at $95 \%$ confidence interval. The mean difference, standard deviation, and standard error were calculated using the STATA 9.0.

\section{Results}

Of the seven patients who passed the inclusion criteria, only six were included in the analysis. The pre-treatment radiographs of one patient were unfit for analysis because they did not exhibit the posteroanterior projection of the left hand which is needed to measure the metacarpal index.

The six patients included in the study consisted of two female and four male patients. Two patients are classified to have OI type IV (moderate) and four are classified to have OI type III. The patient's baseline characteristics are listed in Tables 1 and 2.

There is note of an increase in the metacarpal index in five of the six patients. However, when results are subjected to statistical analysis using a paired t-test with a confidence interval of $95 \%$, the increase in the metacarpal index is not significant (Table 3).

All patients tolerated the infusions and there were no reported adverse reactions to the intravenous bisphosphonate. Only one patient reported a fracture during the six months of treatment (Table 4).

Table 1. Baseline Characteristics of Patients $(n=6)$.

\begin{tabular}{|c|c|c|c|c|c|c|}
\hline Variable & Patient 1 & Patient 2 & Patient 3 & Patient 4 & Patient 5 & Patient 6 \\
\hline Age (years) & 1 yr $10 \mathrm{mos}$ & 8 yrs & 4 yrs & 9 yrs 7 mos & 8 yrs 6 mos & 3 yrs 9 mos \\
\hline Weight (kg) & 5.8 & 8.5 & 7.3 & 12 & 12 & 12.5 \\
\hline Length/Height $(\mathrm{cm})$ & 63 & 60 & 64 & 97.8 & 81 & 92.5 \\
\hline Vitamin D level (mg/ml) & 29.4 & 60 & 16.6 & - & 28.3 & - \\
\hline Seurm calcium (mmol/L) & 2.61 & 2.41 & 2.68 & 2.28 & 2.17 & 2.7 \\
\hline Serum magnesium (mmol/L) & 0.85 & 0.80 & 1.13 & - & 0.91 & - \\
\hline Serum phosphorus (mmol/L) & 1.34 & 1.52 & 1.38 & 1.77 & 1.1 & 1.5 \\
\hline Serum alkaline phosphatase $(\mathrm{mmol} / \mathrm{L})$ & 489 & - & 130.96 & 183 & 333 & 286 \\
\hline Serum BUN (mmol/L) & 1032 & - & 5.13 & 3.82 & 3.4 & 3.69 \\
\hline Serum creatinine (umol/L) & 1 & - & 57.91 & 32 & 33.8 & 12.2 \\
\hline Baseline Metacarpal index (mm) & 0.22 & 0.21 & 0.11 & 0.49 & 0.48 & 0.47 \\
\hline Fractures ( 6 months prior to treatment) & 2 & 1 & 2 & 0 & 0 & 1 \\
\hline
\end{tabular}

Table 2. Consolidated Baseline Characteristics of Patients

\begin{tabular}{|c|c|c|c|c|c|}
\hline Variable & Observed & Mean & Standard Deviation & Minimum & Maximum \\
\hline Age (years) & 6 & 5.958333 & 3.183617 & 1.833333 & 9.75 \\
\hline Weight $(\mathrm{kg})$ & 6 & 9.683333 & 2.994272 & 5.8 & 12.5 \\
\hline Length/Height $(\mathrm{cm})$ & 6 & 76.38333 & 16.37442 & 60 & 97.8 \\
\hline Vitamin D level (mg/ml) & 4 & 33.575 & 18.54443 & 16.6 & 60 \\
\hline Seurm calcium (mmol/L) & 6 & 2.475 & 0.173292 & 2.28 & 2.7 \\
\hline Serum magnesium (mmol/L) & 4 & 0.9225 & 0.154354 & 0.8 & 1.13 \\
\hline Serum phosphorus (mmol/L) & 6 & 1.438333 & 0.271766 & 1.1 & 1.77 \\
\hline Serum alkaline phosphatase $(\mathrm{mmol} / \mathrm{L})$ & 5 & 284.392 & 139.6885 & 130.96 & 489 \\
\hline Serum BUN (mmol/L) & 5 & 3.472 & 1.374216 & 1.32 & 5.13 \\
\hline Serum creatinine (umol/L) & 5 & 27.382 & 20.58943 & 1 & 57.91 \\
\hline Baseline Metacarpal index (mm) & 6 & 0.33 & 0.168879 & 0.11 & 0.49 \\
\hline Fractures per month ( 6 months prior to treatment) & 6 & 0.17 & 0.1520526 & 0 & 0.34 \\
\hline
\end{tabular}

Table 3. Comparison of baseline levels and levels six months after pamidronate therapy.

\begin{tabular}{lccc}
\hline & $\begin{array}{c}\text { Metacarpal index before } \\
\text { therapy }\end{array}$ & $\begin{array}{c}\text { Metacarpal index after } 6 \\
\text { months of therapy }\end{array}$ & Paired t-test \\
\hline $\mathrm{N}$ & 6 & 6 & 6 \\
Mean & 0.3300 & 0.3833 & $0.053(-0.11,0.117)$ p-value $=0.0874$ \\
Standard deviation & 0.1689 & 0.1512 & 0.0612 \\
Standard error of measurement & 0.0689 & 0.0617 & 0.025 \\
\hline
\end{tabular}

Table 4. Comparison of fracture incidence six months prior to initiating pamidronate therapy and while undergoing pamidronate therapy.

\begin{tabular}{|c|c|c|c|c|c|}
\hline Variable & Observed & Mean & Standard Deviation & Minimum & Maximum \\
\hline Fractures per month (6 months prior to treatment) & 6 & 0.17 & 0.1520526 & 0 & 0.34 \\
\hline Fractures per month (during treatment) & 6 & 0.057 & 0.1388044 & 0 & 0.34 \\
\hline
\end{tabular}




\section{Discussion}

Medications used in OI increase bone strength either by increasing the amount of bone protein matrix or by increasing bone mineralization via reduction in bone remodelling. ${ }^{8}$ The use of bisphosphonates, either orally or intravenously, represents a major advance in the treatment of osteoporotic disorders. ${ }^{23}$ Cyclic intravenous pamidronate is currently the most widely used medical therapy for children with moderate to severe OI. ${ }^{2}$

This retrospective analysis of six patients with moderate and severe OI who underwent intravenous pamidronate therapy for six months demonstrated an increase in bone mineral density as evidenced by an increase in the metacarpal index. Although the increase is not statistically significant when subjected to analysis, the increasing trend shows favourable results. The follow-up period of six months may have been quite early to show any statistically significant change. A study by Kusumi et al ${ }^{24}$ have shown statistically significant results in the bone mineral density measured through lumbar spine DEXA among children under 24 months of age with OI receiving pamidronate therapy after one year of treatment.

Similar studies on the use of pamidronate in children have shown an increase in bone mineral density compared to baseline. $8,14-16,23-27$ It must be noted however, that these studies made use of DEXA to measure bone mineral density in the lumbar spine. Due to the expense of this procedure, the patients in this study did not have this done.

A study by Glorieux et al, ${ }^{14}$ reported an increase in the metacarpal width $27 \pm 20.2 \%$ increase per year from baseline following pamdironate infusion every 4-6 month intervals for 1.3-5 years. This study show slightly better results with an increase in the metacarpal index post-treatment by $16 \%$ (in 6 months) compared to the reported 13\% increase (in 6 months). The increased values show an improvement in the patients' osteopenia.

It is noted that in Glorieux et al's study, the pamidronate infusion was given for three consecutive days and overall, the children in their study received a mean of $6.8 \pm 1.1 \mathrm{mg} / \mathrm{kg} /$ year with an initial interval of 6 months between cycles. The six subjects included in the study received a higher dose of pamidronate at $6 \mathrm{mg} / \mathrm{kg}$ over a period of 6 months. They also underwent infusions at shorter intervals (monthly). These differences may account for the slightly better results.

The cyclic administration of intravenous pamidronate reduced the incidence of fracture. ${ }^{1,14,16}$ In this study, four out of the six subjects reported fractures six months prior to therapy. While treatment was on-going, only one patient reported a fracture. Salehpour ${ }^{1}$ cautions that fracture incidence is a weak efficacy parameter because it can be influenced by external factors including mode of handling, and mobility, and may spontaneously decrease with age. Since the subjects in this retrospective review were not compared to the fracture rate in an age-matched control group, it is difficult to ascertain whether the decrease in fracture can be attributed to pamidronate alone.

Despite encouraging results, safety issues are a concern when bisphosphonates are administered to children and adolescents. $^{22}$ Reported side effects include transient flu-like symptoms occurring 12-36 hours post infusion, drop in serum calcium concentrations and other adverse drug reactions. ${ }^{8,22}$ In this study, there are no reported adverse reactions following pamidronate therapy, possibly due to the administration of Ibuprofen prior to starting the pamidronate infusion, as stated in the UP-PGH protocol. ${ }^{20}$ Despite the absence of side effects, however, it is important to continue monitoring these patients during subsequent cycles.

\section{Limitations and Recommendations}

The study only included patients whose pre- and posttreatment radiographic plates were available. This study could have included additional patients but their radiographs were no longer available as x-ray plates older than 5 years old were discarded.

This study shows that although there is an increase in the metacarpal index of the patients, the increase is not statistically significant. It is recommended that the patients be followed up for a longer period of time. Another recommendation is to increase the sample size. Using the computed standard deviation of 0.0612 , a statistically significant change (delta $=0.04$ ) with a $95 \%$ confidence interval may be reached with a population of 12 patients.

The available radiographs were taken using different $x$ ray machines. While bone mineral density calculation was not noticeably affected by changes of film-focus distance, exposure level or film sensitivity/film brand it was influenced by the tube voltage. ${ }^{28}$ Further, it is difficult to define the exact location of the endosteal surface of each metacarpal shaft. Digital x-ray imaging is suggested because this defines the bone edges more accurately, anatomic landmarks are identified automatically, and multiple measurements can be done quickly. ${ }^{13}$

\section{Conclusion}

Overall, the results of this study concurs with the results of previous studies which showed that among patients with osteogenesis imperfecta who received intravenous pamidronate therapy, an increase in bone mineral density and a decrease in fracture rate is expected and that intravenous bisphosphonate therapy is generally well tolerated. 


\section{References}

1. Salehpour Sh, Tavakkoli S. Cyclic pamidronate therapy in children with osteogenesis imperfecta. Int J Endocrinol Metab. 2008; 1:6-12.

2. Cheung MS, Glorieux FH. Osteogenesis Imperfecta: Update on presentation and management. Rev Endocr Metab Disord. 2008; 9(2):153-60.

3. Shapiro JR. Chapter 2 Clinical and Genetic Classification of Osteogenesis Imperfecta and Epidemiology in Shapiro JR, Byers PH, Glorieux FH, Sponseller PD (eds) Osteogenesis Imperfecta: A Translational Approach to Brittle Bone Disease. Elsivier Inc., 2014

4. Chevrel G. Osteogenesis Imperfecta. Orhpanet encyclopedia [Online]. June 2004 [cited 2011 June]. Available from http://www.orpha.net/data/patho/GB/uk-OI.pdf

5. Engelbert RH, Gerver WJ, Breslau-Siderius LJ, et al. Spinal complications in osteogenesis imperfecta: 47 patients 1-16 years of age. Acta Orthop Scand. 1998; 69(3):283-6.

6. Shah I, Johari A. Oral bisphosphonate treatment for osteogenesis imperfecta - an Indian perspective. Ann Trop Pediatr. 2007; 27(1):39-43.

7. Steiner RD, Pepin MG, Byers PH. Osteogenesis Imperfecta. In Pagon RA, Bird TD, Dolan CR, Stephens K, eds. Gene Reviews. University of Washington, Seattle; 1993.

8. Giraud F, Meunier PJ. Effect of cyclical intravenous pamidronate therapy in children with osteogenesis imperfecta. Open-label study in seven patients. Joint Bone Spine. 2002; 69(5):486-90.

9. Russell RG. Bisphosphonates: mode of action and pharmacology. Pediatrics. 2007; 119 Suppl 2:S150-62.

10. Drake MT, Clarke BL, Khosla S., Bisphosphonates: Mechanism of action and role in clinical practice. Mayo Clin Proc. 2008; 83(9):1032-45.

11. Monti E, Mottes M, Fraschini P et al., Current and emerging treatments for the management of osteogenesis imperfect, Ther Clin Risk Manag. 2010; 6:367-81.

12. Dwan K, Philippi CA, Steiner RD, Basel D. Bisphosphonate therapy for osteogenesis imperfecta. Cochrane Database Syst Rev. 2014; 7:CD005088.

13. Biggin A, Munns CF. Osteogenesis Imperfecta: diagnosis and treatment Curr Osteoporos Rep. 2014; 12(3):279-88.

14. Glorieux FH, Bishop NJ, Plotkin $\mathrm{H}$, et al. Cyclic administration of pamidronate in children with severe osteogenesis imperfecta. New Eng J Med. 1998; 339(14):947-52.

15. Falk MJ, Heeger S, Lynch KA, et al. Intravenous bisphosphonate therapy in children with osteogenesis imperfecta. Pediatrics. 2003; 111(3):573-8.
16. Lin $\mathrm{HY}$, Lin SP, Chuang $\mathrm{CK}$, Chen MR, Chang CY. Intravenous pamidronate therapy in Taiwanese patients with osteogenesis imperfecta. Pediatr Neonatol. 2008; 49(5):161-5.

17. Rauch F, Glorieux FH. Osteogenesis Imperfecta. Lancet. 2004; 363(9418):1377-85.

18. Alcausin MMB, de Dios JK, Chiong MD, et al. Intravenous pamidronate treatment in Filipino children with moderate to severe osteogenesis imperfecta. Acta Med Philipp. 2011; 45(4):35-9.

19. Radetti G, Rigon F, Tonini G, et al. Geometry and bone density. Panminerva Med. 2006; 48(3):181-6.

20. Padilla C, De La Paz EC, Chiong MA, et al. Pamidronate Protocol for Osteogenesis Imperfecta (adapted from The Children's Hospital in Westmead), June 2008.

21. Bonnick SL. Bone Densitometry in Clinical Practice, $3^{\text {rd }}$ ed. Humana Press, 2010.

22. Nielsen SP. The metacarpal index revisited: a brief overview. J Clin Densitom. 2001; 4(3):199-207.

23. Shapiro JR, Thompson CB, Wu Y, Nunes M, Gillen C. Bone mineral density and fracture rate in response to intravenous and oral bisphosphonates in adult Osteogenesis Imperfecta. Calcif Tissue Int. 2010; 87(2):120-9.

24. Kusumi K, Ayoob R, Bowden SA, Ingraham S, Mahan JD. Beneficial effects of intravenous pamidronate treatment in children with osteogenesis imperfecta under 24 months of age. J Bone Miner Metab. 2015; 33(5):560-8.

25. Arikoski P, Silverwood B, Tillmann V et al. Intravenous pamidronate treatment in children with moderate to severe osteogensis imperfecta: assessment of indices of dual-energy x-ray absorptiometry and bone metabolic markers during the first year of therapy. Bone. 2004; 34:53946.

26. Forin V, Arabi A, Guigonis V, Filipe G, Bensman A, Roux C. Benefits of pamidronate in children with osteogenesis imperfecta: an open prospective study. Joint Bone Spine. 2005; 72(4):313-8.

27. DiMeglio LA, Peacock M. Two-year clinical trial of oral alendronate versus intravenous pamidronate in children with osteogenesis imperfecta. J Bone Miner Res. 2006; 21(1):132-40.

28. Bottcher J, Pfeil A, Rosholm A, et al. Influence of image-capturing parameters on digital X-ray radiogrammetry. J Clin Densitom. 2005; $8(1): 87-94$

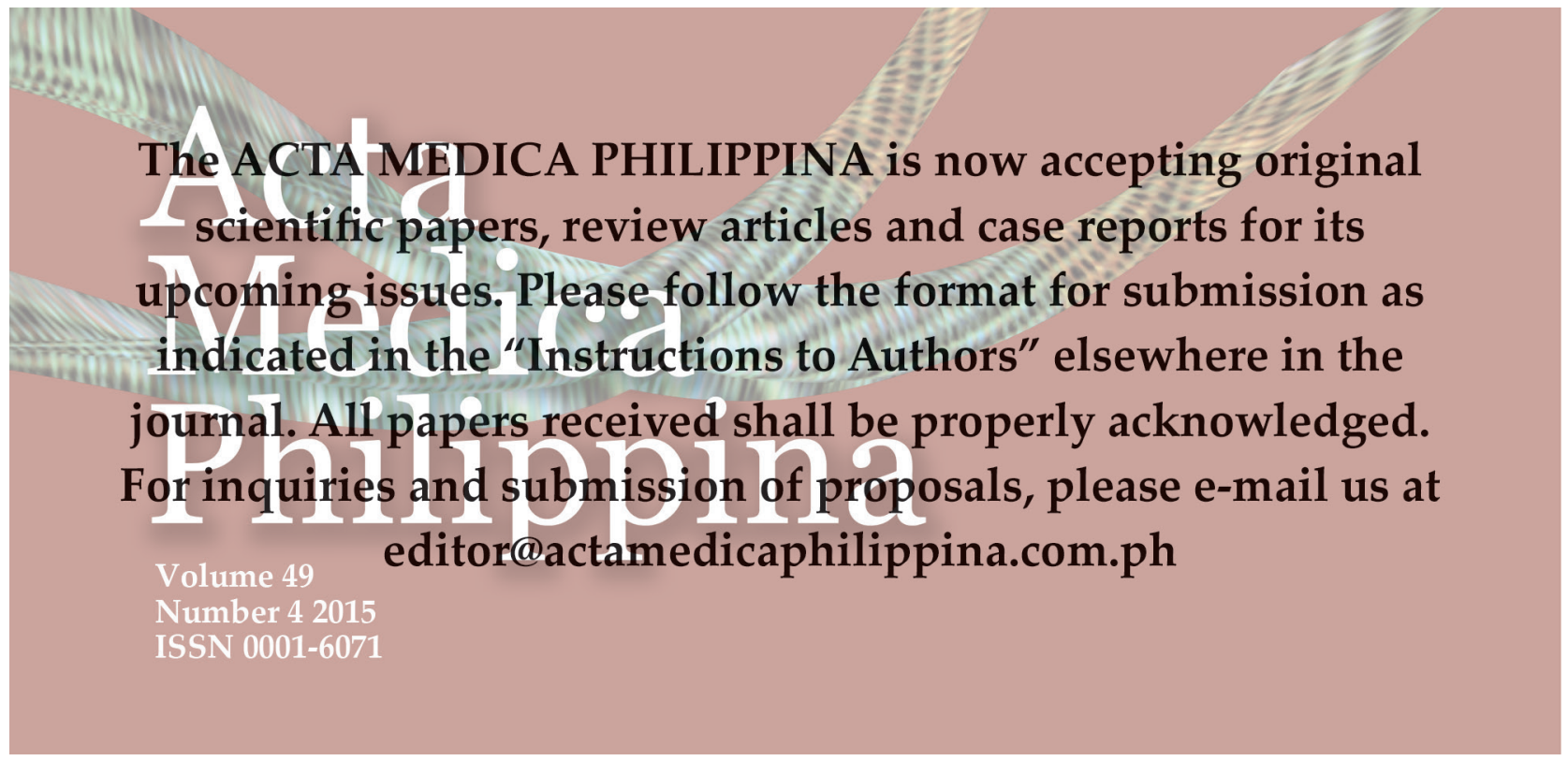

